\title{
Thick Rigidly Foldable Structures Realized by an Offset Panel Technique
}

Bryce Edmondson

Brigham Young University

Robert J. Lang

Lang Origami

Michael R. Morgan

Brigham Young University

Spencer P. Magleby

Brigham Young University - Provo

Larry L. Howell

Brigham Young University - Provo

Follow this and additional works at: https://scholarsarchive.byu.edu/facpub

Part of the Mechanical Engineering Commons

Original Publication Citation

Origami 6, Vol. 1, pp. 149-161

\section{BYU ScholarsArchive Citation}

Edmondson, Bryce; Lang, Robert J.; Morgan, Michael R.; Magleby, Spencer P.; and Howell, Larry L., "Thick Rigidly Foldable Structures Realized by an Offset Panel Technique" (2015). Faculty Publications. 1605. https://scholarsarchive.byu.edu/facpub/1605

This Book Chapter is brought to you for free and open access by BYU ScholarsArchive. It has been accepted for inclusion in Faculty Publications by an authorized administrator of BYU ScholarsArchive. For more information, please contact ellen_amatangelo@byu.edu. 


\title{
Pre-publication version of: \\ Edmondson, B.J., Lang, R.J., Morgan, M.R., Magleby, S.P., Howell, L.L., "Thick Rigidly \\ Foldable Structures Realized by an Offset Panel Technique," Origami 6, American \\ Mathematical Society, Vol. 1, pp. 149-161, 2015.
}

\section{THICK RIGIDLY FOLDABLE STRUCTURES REALIZED BY AN OFFSET PANEL TECHNIQUE}

\begin{abstract}
BRYCE J. EDMONDSON, ROBERT J. LANG, MICHAEL R. MORGAN, SPENCER P.
\end{abstract} MAGLEBY, AND LARRY L. HOWELL

\section{INTRODUCTION}

Rigid-panel origami is often mathematically modeled with idealized zero-thickness panels. When paper is used to realize an origami design, the zero-thickness models are a good approximation. However, many origami-inspired designs require the use of thicker materials that likely will not behave as the zero-thickness kinematic models predict.

The offset panel technique defined previously by the authors Edmondson et al. 14 maintains the kinematics of a zero-thickness origami source model over its full range of motion. The offset panel technique accommodates uniform and varying panel thickness as well as offset panels or gaps between panels. The preserved kinematic behavior allows designers to select an origami model based on desired motion and instantiate it in thick materials.

In this work, we review the offset panel technique and illustrate its capabilities and limitations through several example hardware demonstrations. The examples in the paper are based on the rigidly foldable M3V twist 1 shown in Figure 1. This twist tessellation was developed using the method of fold-angle multipliers Evans et al. 15.

\section{BACKGROUND}

Action Origami. Action origami is a subset of origami of special interest because its mechanisms can be applied to engineering problems. In action origami, some models require deformation of their panels to enable action, one example being the traditional flapping bird Shafer 01, Shafer 10, Lang 11, Lang 97. Others achieve their motion purely through rotation about the folds without the bending of panels. Such models are called "rigidly foldable origami" or "kinematic origami" Bowen et al. 13 .

Modeling and Kinematics. Kinematic origami may be modeled as a network of spherical mechanisms where panels are links and folds are joints Greenberg et al. 11 and can be analyzed using spherical kinematics theory. Each vertex within the structure is modeled as a spherical kinematic mechanism. Spherical kinematics is a

Date: July 31, 2014.

Key words and phrases. Thick Origami, Rigidly Foldable, Origami-inspired, Thickness Accommodation.

This work was funded by the National Science Foundation and Air Force Office of Scientific Research under Grant \#1240417.

1 "M3V" (or $M^{3} V$ ) refers to the crease assignment around the central polygon of the structure: three mountains and one valley fold. 


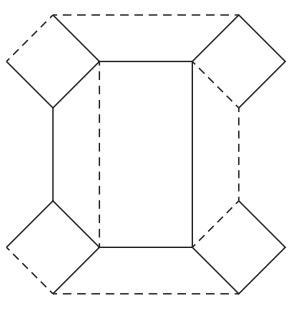

(a)

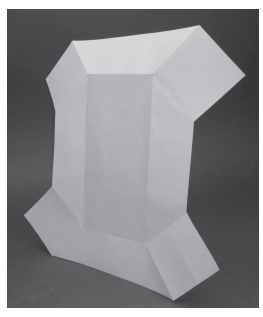

(b)

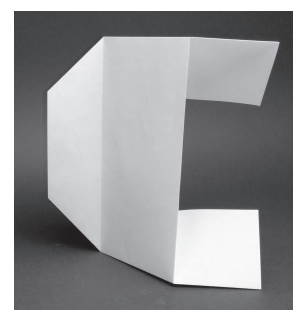

(c)

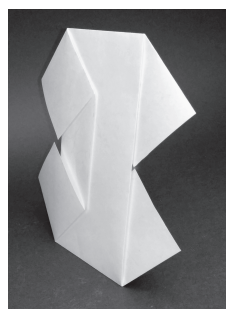

(d)

Figure 1. This origami model is a unit from a M3V twist tessellation. (a) Crease pattern (b) Open position (c) Mid position (d) Closed position

subset of 3-dimensional kinematics where any given point on the mechanism is constrained to move on a spherical surface and all joint axes, whether fixed or instantaneous, intersect at the spherical center. Spherical kinematic motion is the behavior of the rotational axes about the spherical center Chiang 00, Bowen et al. 14]. The spherical kinematics of a model can be preserved as long as the rotational axes' locations and behaviors remain constant even if link size, shape, or both are altered.

Deployable Structures. One potential area of application for origami-inspired design is deployable structures. Deployable structures often use a repeating pattern of coupled mechanisms Gan and Pellegrino 03, such as the Bricard linkage Chen et al. 05] or the Bennett linkage Chen and You 05] to create large singledegree-of-freedom (DOF) mechanisms. Deployable structures are often classified as highly over-constrained mechanisms because the Kutzbach criterion would calculate zero (or negative) DOF, yet they have one DOF due their highly coupled construction Mavroidis and Roth 95a, Mavroidis and Roth 95b.

Thickness Accommodation. In engineering applications of origami-inspired design, accommodation of material thickness is frequently necessary to achieve the design's objective. Existing methods for thickness accommodation can be grouped into two categories: first, methods that preserve range of motion, and second, methods that preserve kinematics. Figure 2 shows a side-by-side illustration of some of the methods described below using a simple four-panel accordion fold.

The axis-shift method [Tachi 11] maintains the range of motion of an origami source model. This method allows the panels to fold by shifting all joints' rotational axes from the center plane to the panel edges (see Figure 2b). Interior degree-4 vertices fold such that there are 2 inside and 2 outside panels. The inside panels fit within the outside panels in thin materials but not in thick materials. A drawback is that in many origami patterns of interest, the vertical offsets break the kinematic motion of the individual vertices.

The offset joint method Hoberman 10 is related to the axis shift method in that each hinge is positioned at the edge of the material (see Figure 2b). The panels are not restricted to be planar, coplanar, or uniform thickness. By extending the hinges away from the panels, gaps are created to allow interior vertices full range of motion, tucking the inside panel into the gap created by the offset. Fully compact cubic bundles were created using this method that can fold and unfold sequentially rather 


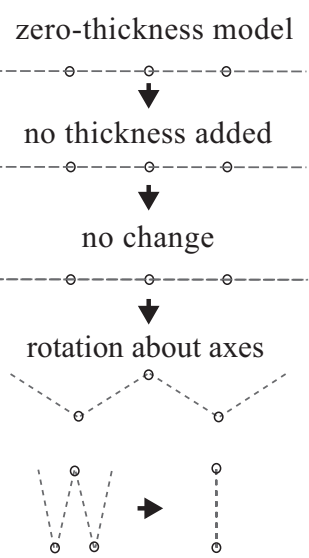

(a)
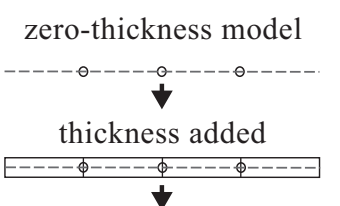

shift rotational axes to edge

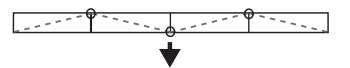

rotation about axes

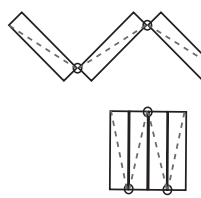

zero-thickness model

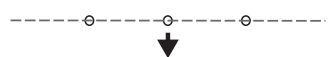

thickness added on membrane

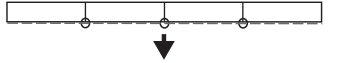

add gaps for valley folds

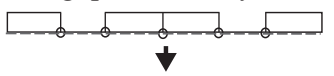

rotation about axes

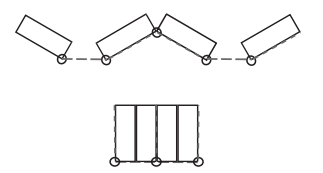

(b)

(c)

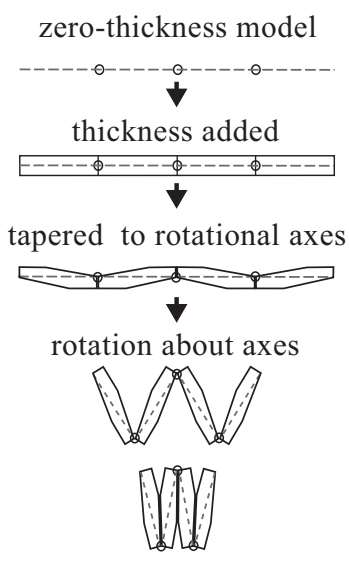

(d)

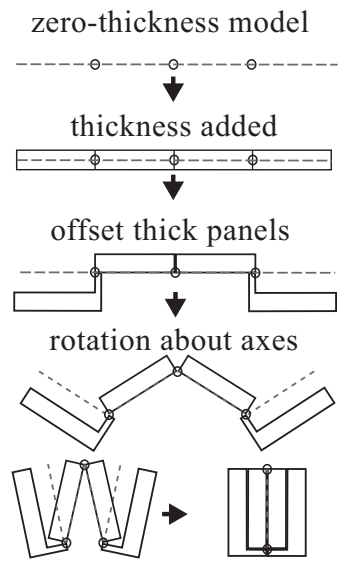

(e)

Figure 2. (a) The zero-thickness model describes the fundamental kinematic behavior. (b) The axis-shift alters the kinematics but can be folded fully compact Tachi 11. The offset joint method results in an identical structure due to the simplicity of this example. (c) The membrane folds method alters the kinematics but can be folded fully compact. (d) The tapered panels method with limited range of motion [Tachi 11. (e) The offset panel technique has the same kinematics as the zero-thickness model and has full range of motion. 
than with preserved kinematics. By utilizing symmetric single parameter vertices, single-DOF mechanisms can be created in thick material using this method.

In the membrane folds method [irbel et al. 13, all rigid panels are attached to one side of a flexible membrane as shown in Figure 2c By controlling the spacing between adjacent panels, full range of motion folding is enabled. Zero gap between panels only allows a mountain fold where a larger gap is necessary for a valley fold with gap width set by panel thicknesses and desired max rotational angle. This gap also provides extra DOFs with small additional motions that can allow a theoretically non-rigidly foldable structure to fold up in practice, however, it can permit undesirable (and unpredictable) additional motions in the deployment.

The tapered panel method Tachi 11] is designed to preserve origami source model kinematics. The panels are trimmed until the panel edges are coincident with the plane defined by the zero-thickness model (see Figure 2d). Because the rotational axes are unchanged, the thick panels' kinematics are equivalent to that of the zero-thickness model. The tapered panel technique, however, yields models that may not be foldable to a fully compact state, and typically do not achieve the full range of motion of the zero-thickness model.

The offset panel technique Edmondson et al. 14] can preserve the kinematics and full range of motion of the origami source mechanism, thus enabling origamiinspired designs to more closely mimic properties identified in zero-thickness models (see Figure 2e).

\section{Offeset Panel Technique}

In rigid origami, panels (facets) can be treated as links and folds as joints Wang and Chen 11, Balkcom and Mason 08, Schenk and Guest 11. Origami mechanisms can be treated as zero-thickness spherical mechanisms, which are mechanisms whose links and joints all lie in a plane in at least one position and whose links are idealized with zero-thickness. In the offset panel technique, the source model's panels are shaped and thickened while maintaining the zero-thickness spherical mechanisms' joint relationships.

The key concept of the technique is that in the fully folded state, all joints lie in a common plane even if one or both panels incident to any joint are spatially offset from that plane, which we refer to as the joint plane. This requirement allows the thick origami mechanism's behavior to be kinematically equivalent to the zero-thickness origami source model, aside from considerations of self-intersections, which must be addressed separately. We accomplish this requirement by creating extensions that connect each panel, whatever its position, with the joint in the joint plane.

Instructions for implementing this technique are reviewed below and include stepby-step examples based on the rigidly foldable M3V twist mechanism. A designbased representation of the steps is provided in Figure 3 .

Step 1. Model Selection. Select an origami source model that gives the desired motion and/or form. The source model must be rigid/flat-foldable. When panels are constrained to be parallel to one another in the folded state, the layer ordering graph on the facets of the source model (a directed graph indicating relative facet order) must be sortable.

Example: We chose the M3V twist shown in Figure1. 


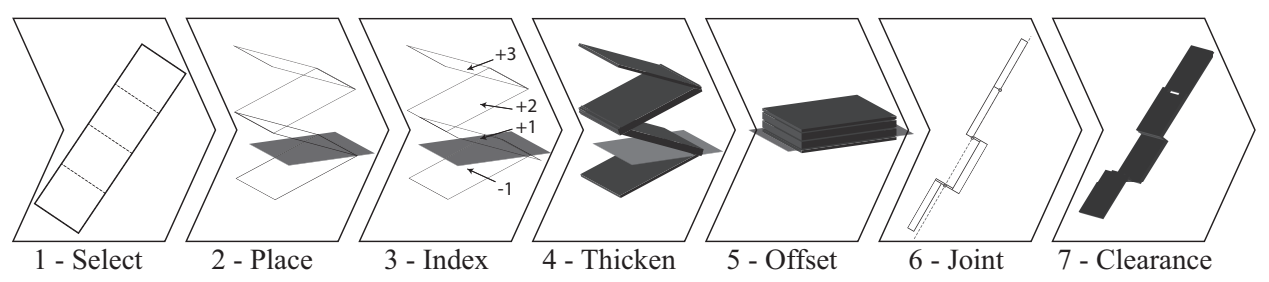

Figure 3. There are 7 steps to design a thick origami model that preserves the kinematics and allows full range of motion using the offset panel technique. Design protocol is followed from the closed position, partially folded views are used here to distinguish between panels for concept visualization.

Step 2. Place Joint Plane. Choose the location of the joint plane, the plane within which all of the joints will lie. Although it is not a requirement, the design is often simplified by assigning the joint plane to be parallel to the panels or even coplanar with the face of one of the panels.

Example: We chose the joint plane to be the center of the closed panels (see Figure 4a. This minimizes the offset distances, which will reduce the potential for self-intersection during the folding motion.

Step 3. Index Panels. Assign each panel an index according to its position relative to the joint plane in the closed state. The joint plane is designated as "0", the panel directly above it as " +1 ", below it as " 1 " and so on.

Example: Figure 4b shows the joint plane in the center of the indexed layers.

Step 4. Thicken Panels. Assign thickness to each panel based on the application.

Example: We assigned a uniform thickness of $3 \mathrm{~mm}$.

Step 5. Offset Panels. Arrange the panels into the closed state according to the indices assigned in step 3. They can be stacked panel to panel or spaced with gaps between panels.

Example: Figure 5a shows the thickened panels stacked in indexed layers.

Step 6. Determine Joints. Extend each joint from the offset position of each panel to the joint plane using rigid extensions so that the axis of rotation lies in the joint plane even if both panels are offset from it Edmondson et al. 14. This ensures that the rotational axes remain unchanged from those of the zero-thickness model throughout the folding motion.

Example: Figure $5 \mathrm{~b}$ illustrates the joint alignment on the crease pattern and sample joint extension configurations. The extensions are rigidly attached to panels with length equal to the panel offset such that the rotational axis remains in the joint plane even if one or both panels are offset.

Step 7. Address Self-Intersection. To prevent panel-joint interference, create clearance holes in panels that lie between another panel's joint and the joint plane. These holes guarantee that the mechanism can be assembled in the fully closed position. These clearance holes are necessary, but not sufficient to guarantee that the mechanism is able to move throughout the entirety of its range of motion. To guarantee full range of motion with no self-intersection, the entire mechanism volume needs to be mapped through the full motion, removing any intersecting 


\section{Closed Top View}

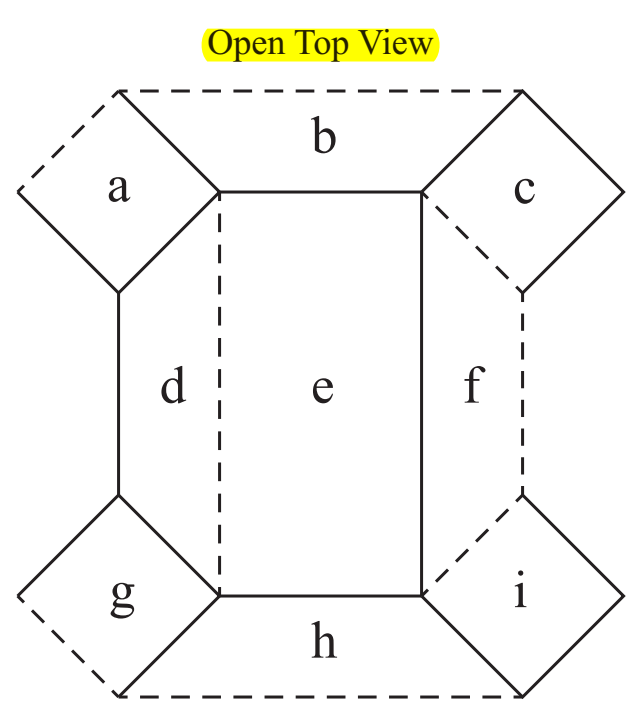

(a)

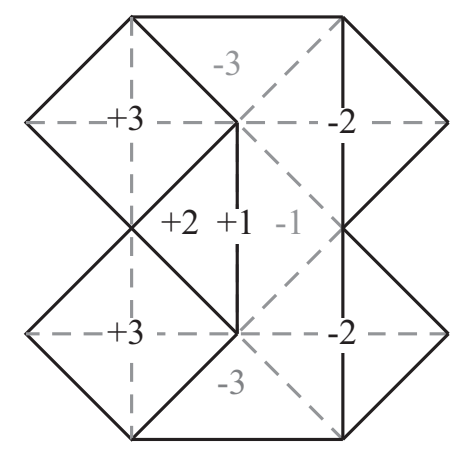

Closed Side View

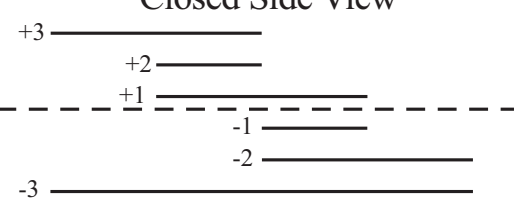

(b)

Figure 4. An illustration of (a) Each panel is labeled with a unique letter on the crease pattern. (b) Step 2 - Place Joint Plane and Step 3 - Index Panels. Side view is shown to illustrate index sequence. Figure 5a further clarifies panel letter/sequence relationship. Solid lines represent panels and the dashed line represents the joint plane in the side view.

material from one or both interfering surfaces. In many cases the clearance holes in the fully closed configuration will be sufficient to avoid self-intersection through the full range of motion.

Example: Clearance holes are located by drawing clearance boundaries around joints and reflecting them about fold lines to their positions on the interfering panels as shown by shaded areas in Figure 6. This process was performed manually in these simple cases but more complex cases could be calculated numerically.

\section{General Examples}

We present several configurations of the offset panel technique that are kinematically equivalent to the zero-thickness structure. The following configurations are not an exhaustive list, but they illustrate some of the capabilities and limitations of the offset panel technique.

The models were created using $3 \mathrm{~mm}$ acrylic sheet stock and approximated joints using adhesive fabric tape to create hinges with minimal play. Each configuration is a version that is kinematically equivalent to the zero-thickness approximation paper model shown in Figure 1 .

Uniform Thickness Panels. The simplest configuration demonstrates uniform panel thickness. Figure 7 shows the M3V twist of Figure 1 in $3 \mathrm{~mm}$ thick panels. 

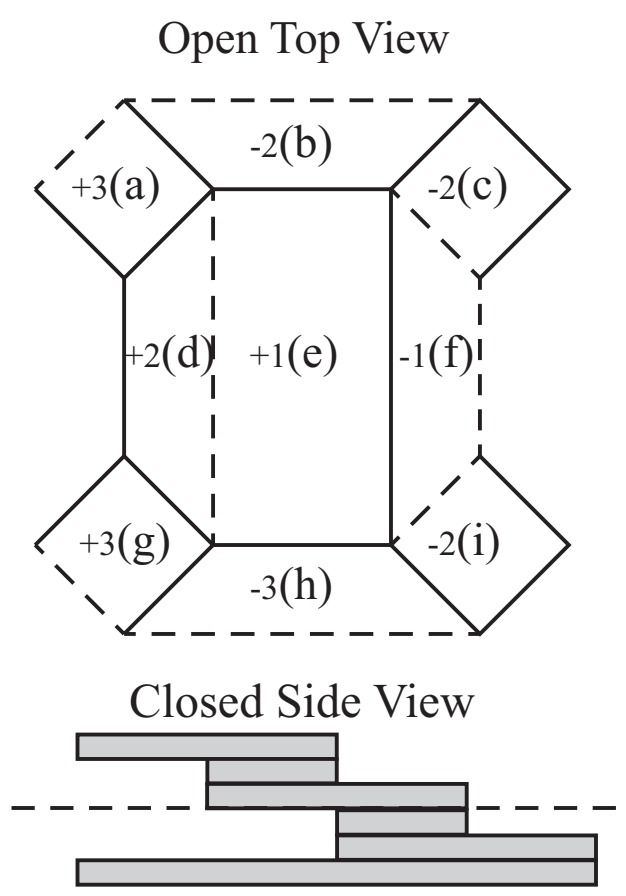

(a)

\section{Open Top View}

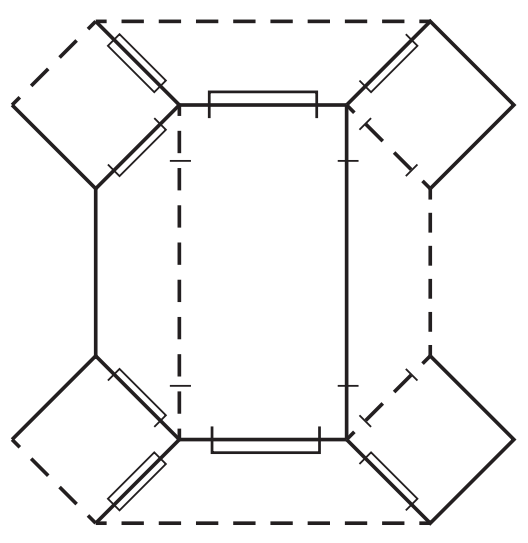

Example Joint Side Views
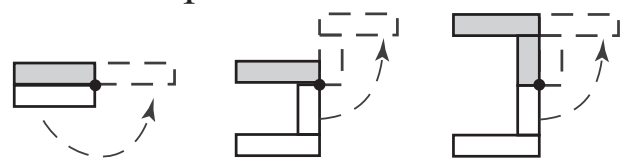

(b)

Figure 5. (a) An open top view with index labels is shown for reference (compare to Figure 4). Steps 4 - Thicken Panels and Step 5 - Offset Panels. Shaded rectangles represent thickened panels. (b) Step 6 - Determine Joints is shown. The example joint side views show a non-comprehensive sample of isolated individual joints and their adjacent panels.

By placing the joint plane at the center of the model and offsetting panels to either side, the sum of the distances from panels to the joint plane is minimized, which, in turn, minimizes the number and size of the required clearance holes. Each panel offset is determined by the sum of thicknesses of the panels that lie between that panel and the joint plane.

Offset Joint Plane. It may be desirable to have relatively large distances between panels in the open state. This can be achieved by offsetting all of the panels to one side of the joint plane. The location of the joint plane will affect the positioning of the panels as well as the volume swept by panels in motion. The joint plane is not restricted to a panel's face. Figure 8 shows a model with all panels on one side of the joint plane. Each panel offset is equal to the sum of thicknesses of the panels between that panel and the joint plane plus the offset distance.

Gaps Between Panels. The previous models had no spacing between panels in the closed state. However, gaps between panels could be beneficial, for example, in a folded wiring board to provide clearance for surface-mounted devices that extend above/below the panels. By offsetting the panels such that spaces exist between them, a model with gaps is created (Figure 91. The offset distances are 


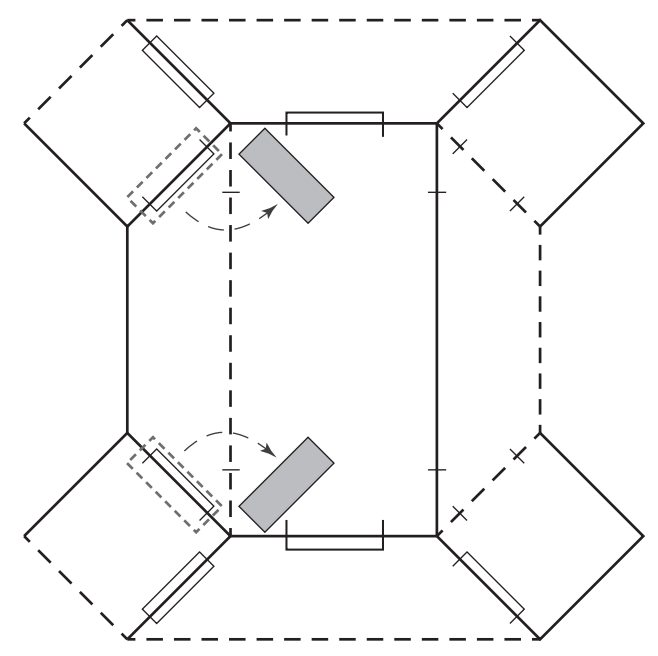

Figure 6. An illustration of Step 7 - Address Self-Intersection. Shaded areas indicate clearance holes located by reflecting a clearance boundary around joints to interferring panels.

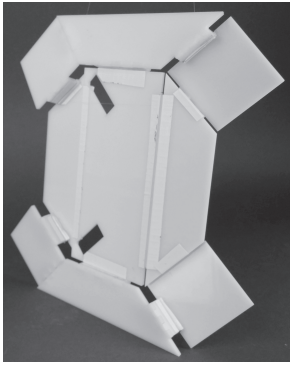

(a)

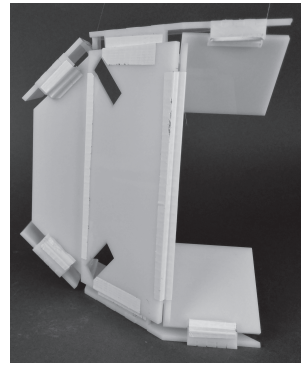

(b)

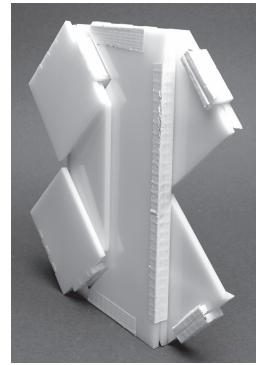

(c)

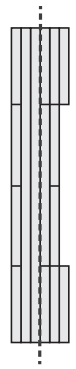

(d)

Figure 7. Uniform thickness model shows the M3V twist in a open, (b) midway, and (c) closed positions. (d) A side view with the joint plane at the dashed line.

now determined by the same sum of panel thicknesses plus the sum of the gaps between the panel and the joint plane.

Arbitrary Uniform Thickness Panels. The offset panel technique accommodates any thickness. Figure 10 shows the same model as Figure 7 with panel thickness four times the previous model's thickness. The farther the panels are offset from the joint plane due to joint plane location or thickness of interior panels, the larger the volume that is swept by the panel and joint, which generally requires increased self-intersection clearance.

Variable Thickness Panels. Different panels can have different thicknesses, not all applications require that all mechanical system panels be equal thickness. Figure 11 shows the example model with panel thicknesses ranging from $3 \mathrm{~mm}$ to $12 \mathrm{~mm}$. 


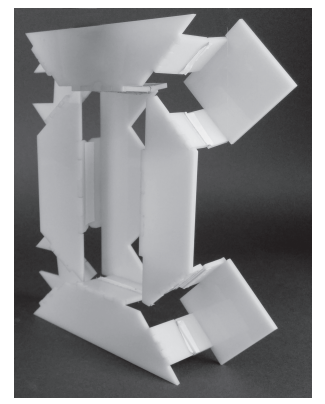

(a)

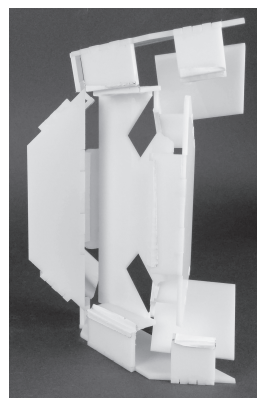

(b)

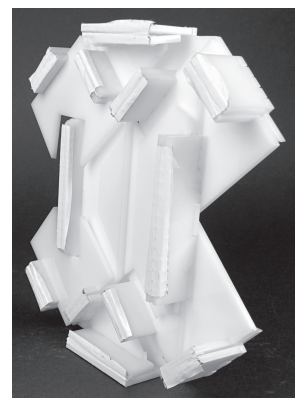

(c)

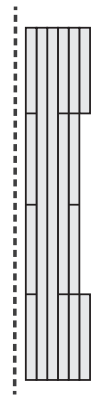

(d)

Figure 8. Far offset model is made with all panels offset to one side of the joint plane in the closed position. This results in the panels spaced far apart in the open position and requires more clearance holes due to more interfering panels. (a) Open, (b) midway, and (c) closed positions shown. (d) A side view with the joint plane at the dashed line.

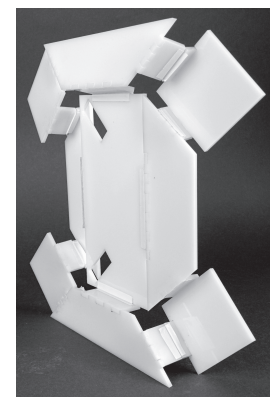

(a)

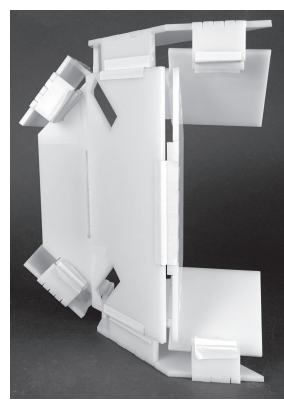

(b)

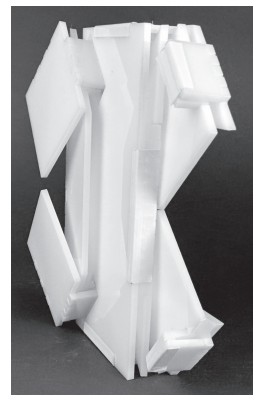

(c)

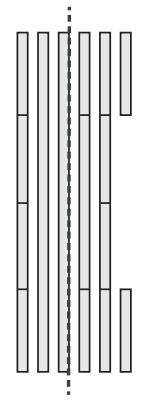

(d)

Figure 9. This model is designed with panels that are spaced apart in the closed position. This has similar open position as the fully compact model but with greater offset distances between panels. (a) Open, (b) midway, and (c) closed positions shown. (d) A side view with the joint plane at the dashed line.

Morphing Volumes. Panels do not have to be sheet-like. As long as the relative joint positions remain fixed within the model, any panel can take on any 3D shape, as long as intersections are avoided during deployment. This allows the creation of a structure with one unique shape when closed that morphs into a different shape when opened.

In Figure 12 we show one such example. We begin with a split-diagonal MVMV twist (adding a fold across the diagonal of the square twist to create a rigidly foldable structure), using the crease pattern shown in Figure 12a. We cut away the four corners of the crease pattern and erect eight cubes on distinct facets of the pattern and thicken the panels according to the offset panel technique. The result is a structure that, in the folded state, forms a larger cube, but in the unfolded 


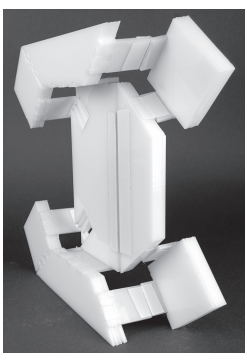

(a)

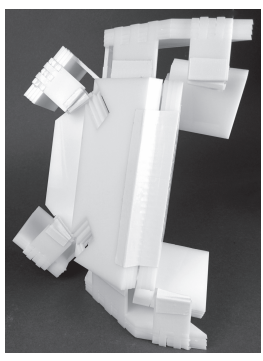

(b)

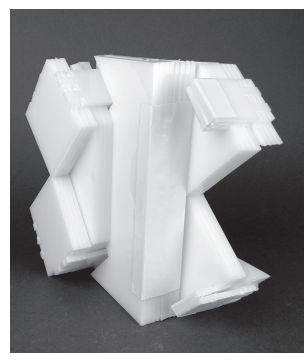

(c)

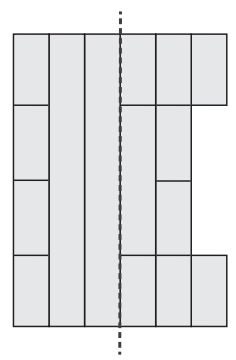

(d)

Figure 10. This model is four times as thick as the initial thick model. The thickness accommodation capability is clearly shown by this very thick model. (a) Open, (b) midway, and (c) closed positions shown. (d) A side view with the joint plane at the dashed line.

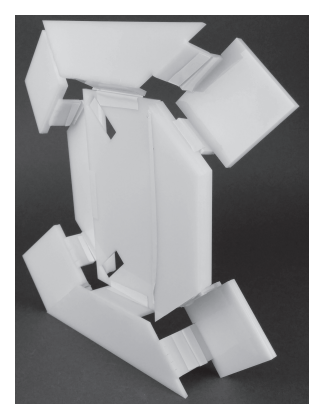

(a)

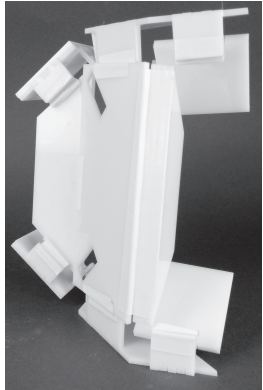

(b)

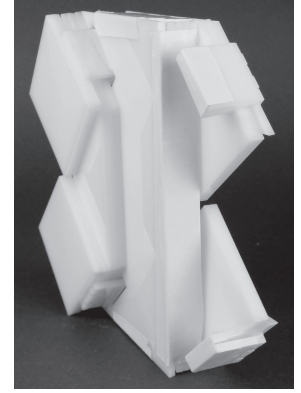

(c)

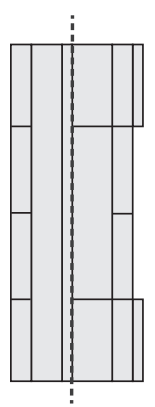

(d)

Figure 11. This model is made from panels all with different thicknesses. (a) Open, (b) midway, and (c) closed positions shown. (d) A side view with the joint plane at the dashed line.

state, takes on a dramatically different shape, with each cube rotating through a unique path throughout the folding motion.

\section{Conclusions}

Using the offset panel technique, one can design a deployable structure using finite-thickness panels while preserving the full range of motion of a zero-thickness idealized origami mechanism. The offset preserves the source model's kinematics so long as the joint rotational axes are unchanged from the zero-thickness model. Preserving these joint relationships guarantees that the kinematics are equivalent, so the link/panel size and shape can change freely. However, self-intersections must be avoided to ensure the full range of motion, generally, by adding clearance holes. Suitable choice of the location of joint plane and the joints themselves can minimize the need for clearance holes and in some cases, eliminate them entirely. 


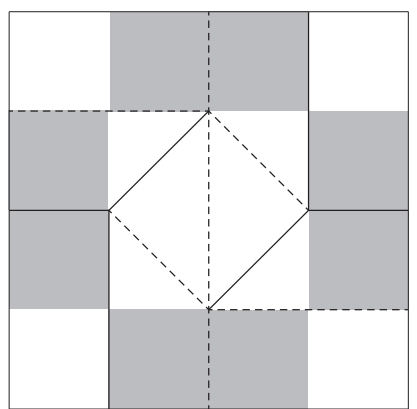

(a)

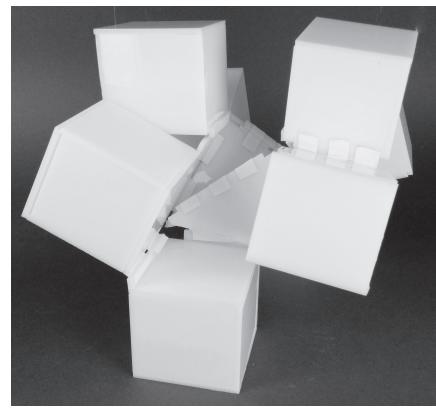

(c)

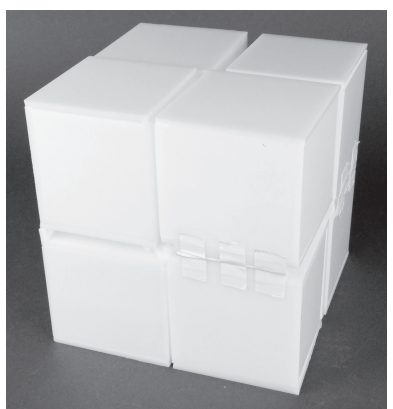

(b)

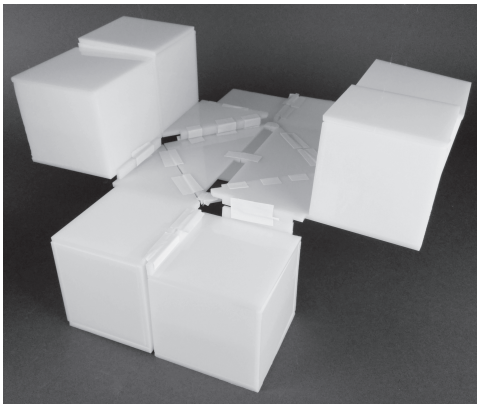

(d)

Figure 12. This MVMV square twist crease pattern a was used to create the above morphing cube. The shaded regions show where the small cubes were added. (b) Open, (c) midway, and (d) closed configurations shown.

Possible small scale applications that could be built on the concepts presented here include packaging, display stands/cases, foldable circuit boards, and solar panels. On a larger scale, the offset panel technique could prove beneficial in morphing architecture, deployable structures, temporary shelters, and deployable solar arrays.

The work in the paper highlights valuable areas of future research that build on the basic technique. These include the development of systematic methods for determining clearances required to avoid self-intersection, modifying motion paths of panels by offsetting the joint plane, and changing the shapes of the panels beyond just adjusting the thickness.

\section{REFERENCES}

[Balkcom and Mason 08] D. Balkcom and M. Mason. "Robotic Origami Folding." The International Journal of Robotics Research 27 (2008), 613-627.

[Bowen et al. 13] Landen A. Bowen, Clayton L. Grames, Spencer P. Magleby, Larry L. Howell, and Robert J. Lang. "A Classification of Action Origami as Systems of Spherical Mechanisms." Journal of Mechanical Design 135:11 (2013), 111008. Available online (http://dx.doi.org/ 10.1115/1.4025379).

[Bowen et al. 14] Landen A. Bowen, Weston Baxter, Spencer P. Magleby, and Larry L. Howell. "A Position Analysis of Coupled Spherical Mechanisms Found in Action Origami." Mechanism and Machine Theory 77 (2014), 13-44.

[Chen and You 05] Yan Chen and Zhong You. "Deployable Structure.", 2005. US 6941704 B2. 
BRYCE J. EDMONDSON, ROBERT J. LANG, MICHAEL R. MORGAN, SPENCER P. MAGLEBY, AND LARRY L. HOWELL

[Chen et al. 05] Yan Chen, Zhong You, and Tibor Tarnai. "Threefold-symmetric Bricard linkages for deployable structures." International Journal of Solids and Structures 42:8 (2005), 22872301. Available online (http://dx.doi.org/10.1016/j.ijsolstr.2004.09.014).

[Chiang 00] C. H. Chiang. "Kinematics of Spherical Mechanisms.", 2000.

[Edmondson et al. 14] Bryce J. Edmondson, Robert J. Lang, Spencer P. Magleby, and Larry L. Howell. "An Offset Panel Technique for Rigidly Foldable Origami." Proceedings of ASME IDETC/CIE 20142014.

[Evans et al. 15] Thomas A. Evans, Robert J. Lang, Spencer P. Magleby, and Larry L. Howell. "Rigidly Foldable Origami Twists." In Origami 6: Sixth International Meeting of Origami in Science, Mathematics, and Education. AMS, 2015.

[Gan and Pellegrino 03] WW Gan and Sergio Pellegrino. "Closed-loop deployable structures." In Proceedings of 44th AIAA/ASME/ASCE/AHS/ASC structures, structural dynamics, and materials conference, Norfolk, pp. 7-10, 2003.

[Greenberg et al. 11] H. Greenberg, M. Gong, S. Magleby, and L. Howell. "Indentifying links between origami and compliant mechanisms." Mechanical Sciences 2 (2011), 217-225.

[Hoberman 10] Charles Hoberman. "Folding Structures Made of Thick Hinged Panels.", 2010. US 7794019.

[Lang 97] R. Lang. Origami in Action : Paper Toys That Fly, Flap, Gobble, and Inflate. St. Martin's Griffin, 1997.

[Lang 11] Robert J. Lang. Origami Design Secrets: Mathematical Methods for an Ancient Art, Second Edition. A K Peters/CRC Press, 2011.

[Mavroidis and Roth 95a] C. Mavroidis and B. Roth. "Analysis of Overconstrained Mechanisms." Journal of Mechanical Design 117 (1995), 69-74.

[Mavroidis and Roth 95b] C. Mavroidis and B. Roth. "New and Revised Overconstrained Mechanisms." Journal of Mechanical Design 117 (1995), 75-82.

[Schenk and Guest 11] M. Schenk and S. Guest. "Origami Folding: A Structural Engineering Approach." In Origami 5: Fifth International Meeting of Origami Science, Mathematics, and Education, , edited by P. Wang-Iverson, R. Lang, and M. Yim, , pp. 291-304. CRC Press, 2011.

[Shafer 01] J. Shafer. Origami to Astonish and Amuse. St. Martin's Griffin, 2001.

[Shafer 10] J. Shafer. Origami Ooh La La! Action Origami for Performance and Play. CreateSpace Independent Publishing Platform, 2010.

[Tachi 11] T. Tachi. "Rigid-Foldable Thick Origami." In Origami 5: Fifth International Meeting of Origami Science, Mathematics, and Education, , edited by P. Wang-Iverson, R. Lang, and M. Yim, , pp. 253-264. CRC Press, 2011.

[Wang and Chen 11] K. Wang and Y. Chen. "Folding a Patterned Cylinder by Rigid Origami." In Origami 5: Fifth International Meeting of Origami Science, Mathematics, and Education, , edited by P. Wang-Iverson, R. Lang, and M. Yim, , pp. 265-276. CRC Press, 2011.

[Zirbel et al. 13] Shannon A. Zirbel, Robert J. Lang, Mark W. Thomson, Deborah A. Sigel, Phillip E. Walkemeyer, Brian P. Trease, Spencer P. Magleby, and Larry L. Howell. "Accommodating Thickness in Origami-Based Deployable Arrays 1." Journal of Mechanical Design 135:11 (2013), 111005. Available online (http://dx.doi.org/10.1115/1.4025372).

Dep. of Mechanical Engineering, Brigham Young University, Provo, Utah 84602

E-mail address: edmondbr@byu.edu

Lang Origami, Alamo, CA 94507

E-mail address: robert@langorigami.com

Dep. of Mechanical Engineering, Brigham Young University, Provo, Utah 84602

E-mail address: morgan44@byu.edu

Dep. of Mechanical Engineering, Brigham Young University, Provo, Utah 84602

E-mail address: magleby@byu.edu

Dep. of Mechanical Engineering, Brigham Young University, Provo, Utah 84602

E-mail address: lhowell@byu.edu 